\title{
DENSIDADE DA FAUNA INVERTEBRADA DA SERAPILHEIRA EM FUNÇÃO DO EFEITO DE BORDA DE DIFERENTES ÁREAS DE REFLORESTAMENTO
}

\footnotetext{
Raissa Nascimento dos Santos ${ }^{1}$, Wilbert Valkinir Cabreira ${ }^{2}$

1 Mestranda em Ciências Ambientais e Florestais (nsantos.raissa@gmail.com), Universidade Federal Rural do Rio de Janeiro, Departamento de Silvicultura, Seropédica, Rio de Janeiro, Brasil.

2 Doutorando em Ciências Ambientais e Florestais, Universidade Federal Rural do Rio de Janeiro, Departamento de Silvicultura, Seropédica, Rio de Janeiro, Brasil.

Recebido em: 04/10/2019 - Aprovado em: 30/11/2019 - Publicado em: 15/12/2019 DOI: 10.18677/EnciBio_2019B19
}

\begin{abstract}
RESUMO
O efeito de borda expõe os organismos ali presentes à diferentes condições abióticas provocando alterações na densidade, distribuição, comportamento e interações das espécies. O objetivo deste estudo foi avaliar a variação na densidade da fauna invertebrada presente na serapilheira em relação ao efeito de borda de duas áreas reflorestadas com diferentes idades. Foram alocadas 10 parcelas em cada uma das Trilhas, Marrom (TM) e Amarela (TA), que apresentam tempo de reflorestamento de 11 e cinco anos de implantação respectivamente. Dois gradientes foram escolhidos para amostragem, borda e a 15 metros para o interior das áreas reflorestadas, nas quais foram mensurados a taxa de cobertura do solo, luminosidade, além da profundidade e peso da serapilheira. Dentro de cada parcela foi coletada com auxílio de um gabarito de $25 \times 25 \mathrm{~cm}$ a serapilheira para posterior triagem da fauna invertebrada, tendo sua densidade determinada. Houve maior dissimilaridade da fauna invertebrada no gradiente borda na área com menor tempo de reflorestamento (TA). A taxa de cobertura do solo e profundidade da serapilheira apresentaram alta correlação com a densidade da fauna invertebrada. Áreas com maior idade de reflorestamento reduz o efeito de borda propiciando menor variabilidade da densidade da fauna invertebrada. O grupo Symphyla é indicativo de ambientes com estrato arbóreo bem desenvolvido com baixa intensidade luminosa e elevado grau de preservação. Os grupos Hymenoptera e Heteroptera são indicadores de ambientes de alto grau de luminosidade. A taxa de cobertura do solo e profundidade da serapilheira propicia melhor desenvolvimento da fauna invertebrada da serapilheira.
\end{abstract}

PALAVRAS-CHAVE: Biodiversidade; Liteira; Unidade de Conservação 


\title{
DENSITY OF THE INVERTEBRATE FAUNA IN THE LITTER AS A RESULT OF EDGE EFFECT IN DIFFERENT REFORESTATION AREAS
}

\begin{abstract}
The edge effect exposes the organisms present there to different abiotic conditions causing changes in species density, distribution, behavior and interactions. The objective of this study was to evaluate the variation in density of invertebrate fauna present in litter in relation to the edge effect of two reforested areas with different ages. Ten plots were allocated to each of the Trails, Brown (TM) and Yellow (TA), which present reforestation time of 11 and 5 years of implementation respectively. Two gradients were chosen for sampling, edge and 15 meters inside from the edge to the reforested areas, in which the soil cover rate, luminosity, and the depth and weight of the litter were measured. Within each plot with the aid of a $25 \times 25 \mathrm{~cm}$ template, the litter was collected for subsequent screening of the invertebrate fauna, having its density determined. There was greater dissimilarity of invertebrate fauna in the edge gradient in the area with the shortest reforestation time (TA). Ground cover rate and litter depth are highly correlated with the density of invertebrate fauna. Areas with greater reforestation age reduce the edge effect, providing lower variability of invertebrate fauna density. The Symphyla group is indicative of environments with well developed tree with low light intensity and high degree of preservation. The Hymenoptera and Heteroptera groups are indicators of high light environments. The soil cover rate and litter depth provide better development of the litter invertebrate fauna.
\end{abstract}

KEYWORDS: Biodiversity; Litter; Conservation Unit

\section{INTRODUÇÃO}

O efeito de borda é resultante da interação entre dois ambientes adjacentes e que se encontram separados por uma zona de transição. Essa mudança abrupta expõe os diversos organismos presentes à diferentes condições abióticas como o aumento da temperatura, vento e consequentemente à redução da umidade provocando alterações na densidade, distribuição, comportamento e interações das espécies (MURCIA, 1995). As relações ecológicas encontradas inseridas nesses ambientes atuam diferentemente na manutenção da biodiversidade, o que garante a sobrevivência e perpetuação das espécies (OLIVEIRA et al., 2016). Diversos organismos desempenham papéis de suma importância para a manutenção e qualidade ambiental, abrangendo a relação com as teias tróficas, cuja base estão as raízes, matéria orgânica e a serapilheira (STORK; EGGLETON, 1992; TANT et al., 2015).

A serapilheira apresenta diversas funções como a proteção do solo de agentes erosivos, além de fornecer nutrientes para os organismos no solo e plantas (AMPOORTER et al., 2015; BERUDE et al., 2015). A qualidade química e física além dos agentes biológicos do solo, estão ligados diretamente com a serapilheira que ao fornecer nutrientes ao solo, favorece o desenvolvimento de organismos que atuam no processo de decomposição do material (SILVA et al., 2017).

Em ambientes tropicais, diversos organismos podem ser encontrados no compartimento da serapilheira (ZARDO et al., 2010). A serapilheira constitui um ambiente adequado para a presença desses indivíduos, o microambiente que a fauna invertebrada encontra nesse compartimento apresenta menores oscilações de temperatura aliado com um maior teor de umidade, sendo ainda abrigo contra 
predadores e fonte de alimento, o que garante a sua sobrevivência (MORGADO et al., 2015).

A fauna invertebrada é considerada um importante bioindicador ambiental, devido a dinâmica solo-serapilheira que eles mantêm e que por meio de suas atividades participam do equilíbrio do ecossistema por ocupar diferentes níveis tróficos da cadeia alimentar (BIANCHI et al., 2017). Esses organismos ainda, são os principais responsáveis pela fragmentação da serapilheira (OSSOLA; NYMAN, 2017). Sendo assim, o processo de decomposição é afetado, havendo a liberação de nutrientes da serapilheira, que influencia a taxa de produção das árvores de material vegetal (KITZ et al., 2015).

Foram testados os possíveis efeitos de borda sobre a comunidade dos invertebrados no compartimento serapilheira. Tem-se como hipótese que o efeito de borda e a heterogeneidade das condições ambientais, levam a mudanças na densidade da fauna invertebrada encontrada na serapilheira. Sendo assim, o objetivo deste estudo foi avaliar a densidade da fauna invertebrada da serapilheira em áreas reflorestadas, correlacionando os diferentes grupos taxonômicos da fauna invertebrada com a taxa de cobertura do solo, profundidade da serapilheira, peso da serapilheira e grau de luminosidade.

\section{Área de estudo}

\section{MATERIAL E MÉTODOS}

O estudo foi realizado na Reserva Ecológica do Guapiaçú - REGUA (22²4'S, $\left.42^{\circ} 44^{\prime} \mathrm{W}\right)$ que faz parte da categoria Reserva Particular de Patrimônio Natural (RPPN) que está incluída dentro do sistema de Unidades de Conservação (UCs) e está situada no município de Cachoeiras de Macacu - RJ.

Segundo Altivo e Rodrigues (2017), a REGUA apresenta um dos maiores remanescentes de Mata Atlântica do estado do Rio de Janeiro. Possui áreas protegidas incluindo áreas de propriedades particulares e parceiras que totalizam cerca de 10.639 ha (AZEVEDO et al., 2018). A tipologia florestal encontrada na reserva, é classificada como Floresta Ombrófila Densa com cobertura vegetal da área variando em quatro fitofisionomias sendo: Aluvial, Terras Baixas, Sub-Montana e Montana (AZEVEDO et al., 2018).

O clima da área é marcado por duas estações distintas segundo a classificação de Köppen do tipo Af, tropical com verão chuvoso e inverno seco e

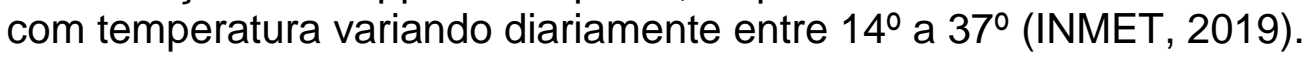

A área amostrada foi composta por duas áreas reflorestadas localizadas nas trilhas Marrom (TM) e Amarela (TA) da reserva e que apresentam diferentes idades de implantação, aproximadamente 11 e cinco anos, respectivamente.

\section{Coleta da serapilheira e triagem da fauna invertebrada}

A coleta foi realizada em julho de 2019. A amostragem da fauna invertebrada do solo, consistiu na utilização de um gabarito de $25 \times 25 \mathrm{~cm}$ no qual cinco parcelas foram alocadas na borda de cada trilha e a 15 metros para o seu interior (Figura 1), totalizando 20 parcelas. 


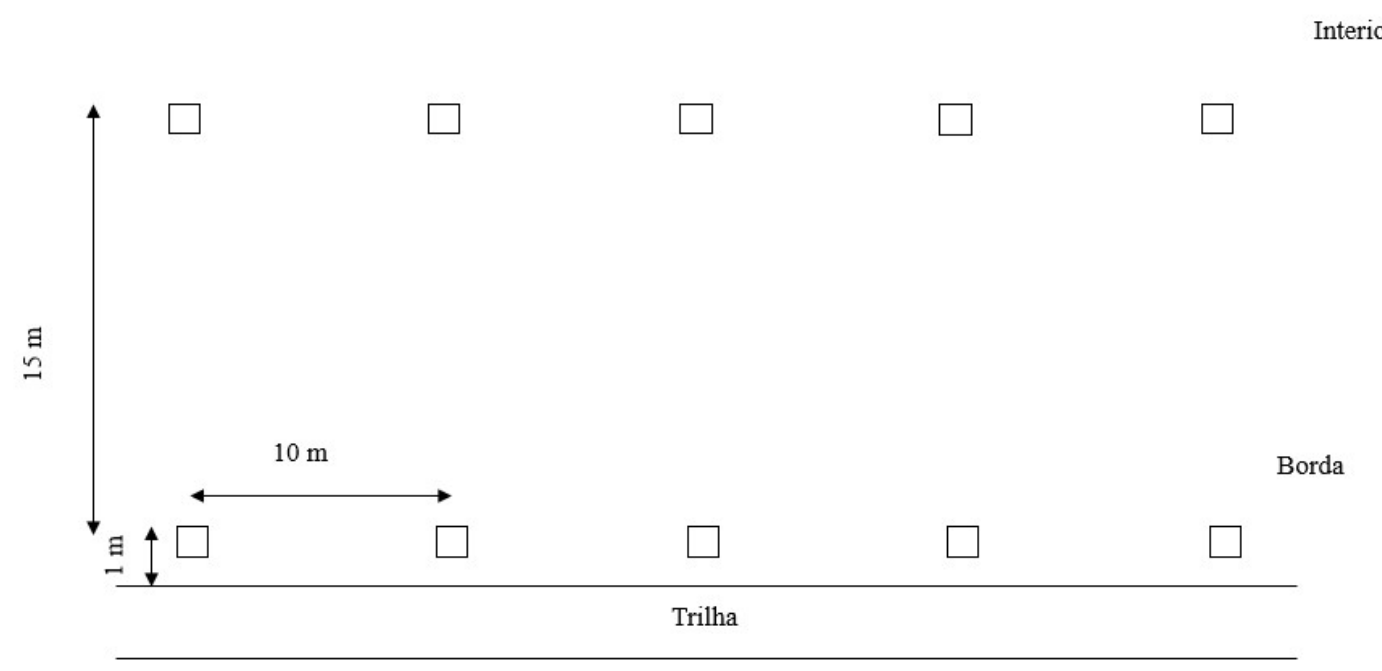

FIGURA 1. Croqui da alocação das parcelas na área de amostragem.

O material vegetal recolhido foi acondicionado em sacos plásticos e transportados para o Laboratório da reserva. Uma primeira triagem foi realizada de forma manual com o auxílio de pinça e lupa de mão para a retirada de organismos da serapilheira. Os indivíduos coletados foram acondicionados em frascos identificados contendo álcool $70 \%$ e com auxílio de uma lupa binocular houve a identificação dos organismos em grandes grupos taxonômicos (classe, ordem, família).

As amostras de serapilheira após terem sido triadas, foram submetidas à secagem em estufa de circulação forçada, a temperatura de $55^{\circ} \mathrm{C}$ por 72 horas. Após esse período, o material foi pesado em balança analítica.

A taxa de cobertura do solo foi estimada visualmente e a profundidade de serapilheira foi mensurada com uma régua graduada antes da retirada da serapilheira da parcela. Uma foto do dossel foi tirada sob as parcelas para quantificar a taxa de cobertura do dossel utilizando-se o aplicativo Canopeo ${ }^{\mathrm{TM}}$ (PATRIGNANI; OCHSNER, 2015), a distância da parcela da borda da trilha para o interior foi medida com auxílio de uma trena.

\section{Análise de dados}

Após a coleta dos dados no campo, foi realizado o cálculo para o grau de luminosidade presente nas parcelas e a densidade da fauna invertebrada (indivíduos $/ \mathrm{m}^{2}$ ). O grau de luminosidade foi quantificado conforme Equação 1.

$$
L=100-\text { TC (1) }
$$

Em que: $L=$ grau de luminosidade (\%); TC = taxa de cobertura do dossel (\%) dado pelo aplicativo Canopeo ${ }^{\mathrm{TM}}$ após anexar a foto.

Foi realizado escalonamento multidimensional não-métrico (N-MDS), utilizando o índice de similaridade de Bray-Curtis como medida de distância, para demonstrar o grau de similaridade da densidade da fauna invertebrada nas áreas reflorestadas. Em seguida, procedeu-se com teste de Bonferroni $(p<0,05)$ para testar a diferença estatística na densidade da fauna invertebrada entre ambos gradientes 
(borda e interior). Para ambas análises, foi utilizado o software estatístico Past $3.0 \mathrm{v}$ 2.17c (HAMMER et al., 2001).

Para verificar as relações entre a densidade dos grupos taxonômicos e os valores da taxa de cobertura do solo, profundidade da serapilheira, peso da serapilheira e luminosidade, foi realizada a análise de componentes principais (ACP) (PEARSON, 1901), utilizando o programa estatístico Minitab.

\section{RESULTADOS E DISCUSSÃO}

O escalonamento multidimensional não-métrico (N-MDS), demonstrou que os dois eixos explicaram $81 \%$ da variabilidade total dos dados do diagrama de ordenação em função da densidade da fauna edáfica, sendo $77 \%$ e $4 \%$ para 0 eixo 1 e 2 respectivamente (Figura 2 ).

A densidade de indivíduos na área reflorestada de 11 anos (TM) não diferiu em função dos gradientes, borda e interior (Figura 2). Contudo na borda da área de cinco anos de reflorestamento, foi observado dissimilaridade com as demais áreas (Figura 2), evidenciando condições mais estáveis para a comunidade da fauna invertebrada em áreas de maior tempo de reflorestamento.

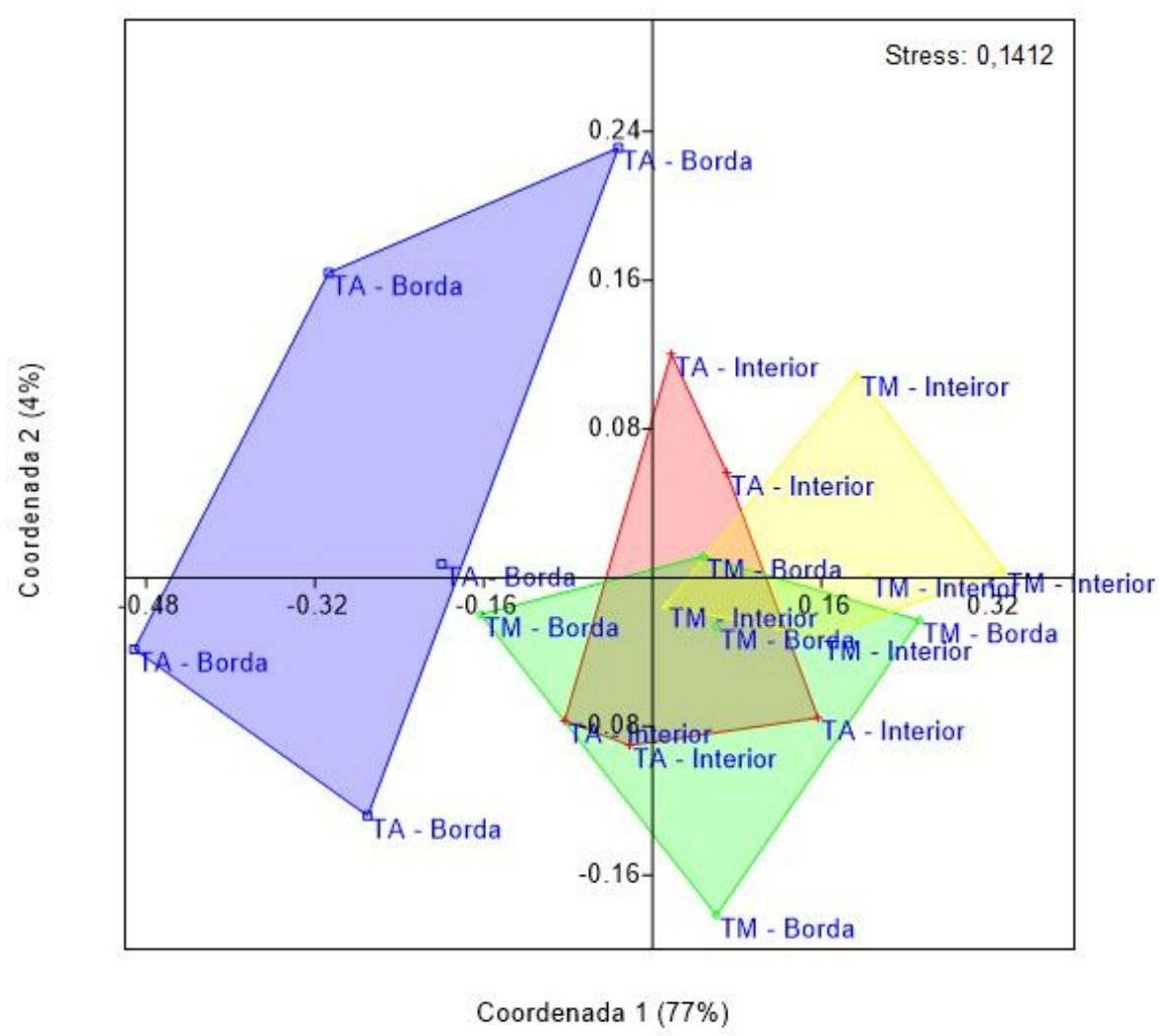

FIGURA 2. Diagrama de ordenação da densidade da fauna invertebrada pelo escalonamento multidimensional não-métrico (N-MDS). TM = área de 11 anos de implantação do reflorestamento. TA = área de cinco anos de implantação do reflorestamento. 
Segundo Silva et al., (2013), as variações ambientais existentes, influenciam na atividade dos grupos da fauna invertebrada e são responsáveis pela menor ou maior correlação dos mesmos em relação a uma área. Em um estudo realizado em fragmentos de Floresta Ombrófila Densa ao avaliarem a fauna invertebrada como possível bioindicadora de preservação, foi constatado que a serapilheira sobre o solo está relacionada diretamente com a atividade da fauna do solo (SCORIZA; CORREIA, 2016).

Os organismos da fauna invertebrada foram distribuídos em 20 grupos taxonômicos. Os grupos que apresentaram maiores valores de densidade média independente da área foram Acari, Collembola, Formicidae e Isopoda (Tabela 1).

TABELA 1. Densidade média (indivíduos $/ \mathrm{m}^{2}$ ) dos grupos taxonômicos da fauna invertebrada nas duas áreas de amostragens, borda e interior de áreas reflorestadas na Reserva Ecológica do Guapiaçu, RJ. TM = área de 11 anos de implantação do reflorestamento. TA = área de cinco anos de implantação do reflorestamento.

\begin{tabular}{|c|c|c|c|c|}
\hline & \multicolumn{2}{|c|}{ TA } & \multicolumn{2}{|c|}{ TM } \\
\hline Grupos taxonômicos & Borda & Interior & Borda & Interior \\
\hline Acari & 32 & 134,4 & 188,8 & 265,6 \\
\hline Aranea & - & 21,33 & 32 & 56 \\
\hline Chilopoda & & 16 & & 16 \\
\hline Coleoptera & 16 & 16 & 16 & 16 \\
\hline Dermaptera & & 16 & & \\
\hline Diplopoda & 24 & 26,66 & 24 & 28 \\
\hline Diptera & 16 & 16 & 16 & 16 \\
\hline Formicidae & 96 & 166,4 & 160 & 355,2 \\
\hline Heteroptera & 16 & 16 & 32 & 16 \\
\hline Hymenoptera & 16 & - & - & - \\
\hline Isopoda & 64 & 150,4 & 140,8 & 217,6 \\
\hline Isoptera & - & 16 & - & - \\
\hline Larva Coleoptera & - & 16 & - & - \\
\hline Larva Lepidoptera & & 16 & - & 32 \\
\hline Orthoptera & 16 & 20 & - & - \\
\hline Pseudoscorpionida & & 16 & - & - \\
\hline Stermorryncha & - & 16 & - & 16 \\
\hline Collembola & 54,4 & 137,6 & 121,6 & 156,8 \\
\hline Opilionidae & - & 16 & 16 & 32 \\
\hline Symphyla & & & & 32 \\
\hline
\end{tabular}

Segundo Almeida et al., (2007) o grupo Acari é influenciado pelo material vegetal aportado sobre o solo e posteriormente pela matéria orgânica proveniente do mesmo. Esse grupo ainda é sensível a umidade, quando se encontram em ambientes com umidade mais alta e menos em ambientes secos (SYLVAIN et al., 2014). Assim, a alta densidade desse grupo na TM principalmente no interior do fragmento pode estar relacionada com a maior deposição de resíduos vegetais sobre o solo decorrente de uma mata mais fechada e antiga.

O grupo Collembola tem como base alimentar, fungos e microrganismos, que estão associados a matéria orgânica e à serapilheira (OLIVEIRA FILHO; BARETTA, 2016), devido a isso, influenciam diretamente na ciclagem de nutrientes e fertilidade dos solos (SCORIZA et al., 2016). Ainda, são responsáveis pelo transporte de carbono da serapilheira para o solo e nas alterações da composição da matéria 
orgânica e o aumento da transferência para a comunidade microbiana do solo (MAAß et al., 2015).

O grupo Formicidae pertencente a ordem Hymenoptera foi um dos grupos que apresentou maior densidade (Tabela 1), uma das razões para elevada quantidade de indivíduos desse grupo pode ser explicada pela resistência das formigas às intempéries e às alterações ambientais em dada área (UNDERWOOD; FISHER, 2006). As formigas têm características biológicas e ecológicas que as tornam sensíveis ao ambiente que vivem e desempenham múltiplos papéis na regulação de processos ecológicos (PETERS et al. 2016). Áreas que apresentem maior complexidade estrutural, como grau de conservação, estratos arbóreos definidos, diversidade de plantas, entre outras, possuem maior disponibilidade de recursos, áreas para nidificação, e consequentemente maior diversidade de formigas (AHUATZIN et al. 2019).

O grupo Isopoda também obteve altos valores de densidade demonstrando que estes grupos que são detritívoros e decompositores atuam preferencialmente no compartimento serapilheira (BARETTA et al., 2010).

O grupo Symphyla foi exclusivo da área de interior do reflorestamento mais antigo (TM), resultados que podem estar associados ao menor grau de luminosidade e maior taxa de cobertura do solo (Figura 3). Souza et al. (2008) afirmam que geralmente esses organismos são encontrados em ambientes que apresenta baixo grau de degradação, evidenciando o elevado grau de preservação da área. A análise de componentes principais, explicou $85,14 \%$ da variabilidade total dos dados $(56,41 \%$ para o eixo 1 e $28,73 \%$ para o eixo 2 ) (Figura 3 ).

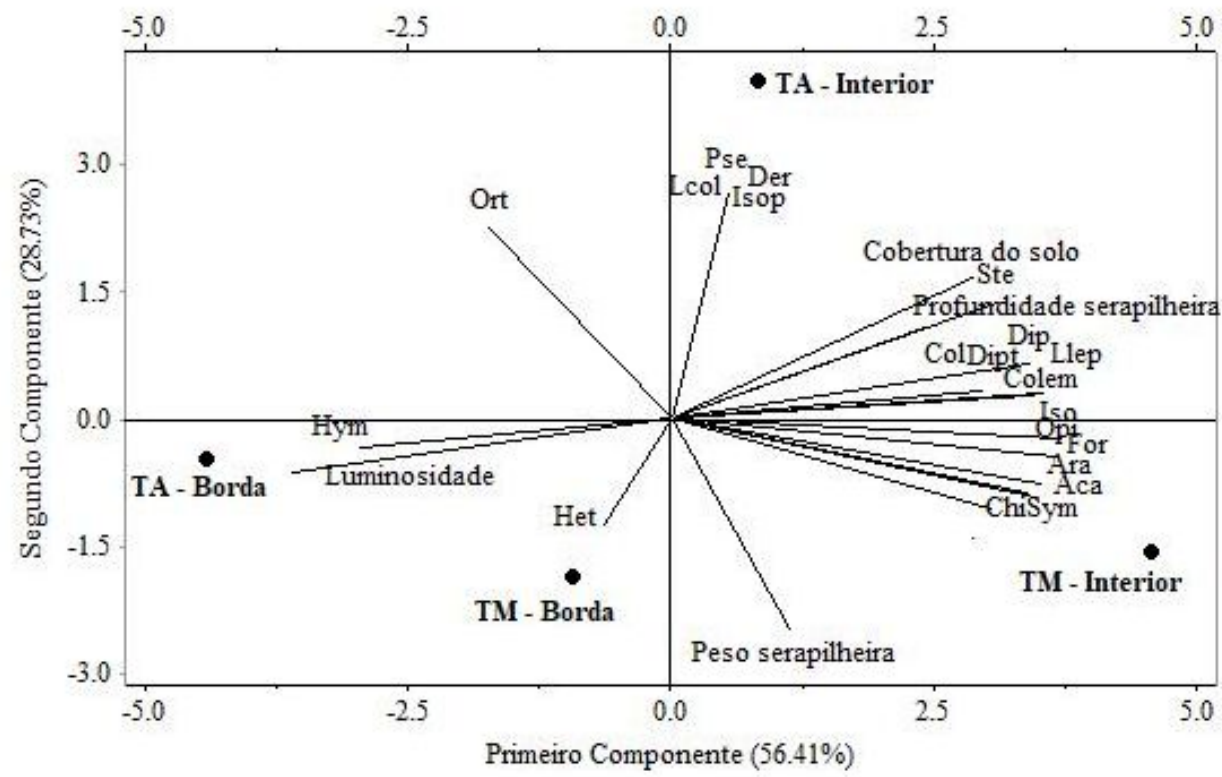

FIGURA 3. Análise de componentes principais da densidade dos grupos taxonômicos da fauna invertebrada do solo, taxa de cobertura do solo, profundidade e peso da serapilheira e luminosidade. Hym: Hymenoptera; Het: Heteroptera; Ort: Orthoptera; Lcol: Larva de coleoptera; Pse: Pseudoscorpionidae; Der: Dermaptera; Isop: Isoptera; Ste: Sternorrhyncha; Dipt: Diptera; Dip: Diplopoda; Llep: Larva de lepidoptera; Colem: Colembolla; Iso: Isopoda; Opi: Opilionidae; For: Formicidae; Ara: Aranaea; Aca: Acari; Sym: Symphyla; Chi: Chilopoda. TM = área de 11 anos de implantação do reflorestamento. TA = área de cinco anos de implantação do reflorestamento. 
Ao longo do eixo 2, verificou-se a separação do gradiente borda em relação ao interior dos fragmentos (Figura 3). Este padrão evidenciou a influência das variáveis mensuradas na distribuição dos grupos taxonômicos da fauna invertebrada. O grau de luminosidade apresentou maior correlação com o gradiente borda, indicando que grupos como Hymenoptera e Heteroptera apresentam preferência à ambientes abertos com maior grau de luminosidade. Já a taxa de cobertura do solo e profundidade da serapilheira apresentaram maior correlação com o gradiente interior. A grande maioria dos grupos taxonômicos apresentaram correlação.

O microclima formado no interior da camada de serapilheira, pode influenciar em maior ou menor afinidade dos grupos da fauna invertebrada a este compartimento (KALU et al., 2015). Diversos grupos apresentaram correlação com a profundidade da serapilheira e cobertura do solo, variáveis que garantem a manutenção da umidade no solo sob a camada de serapilheira, favorecendo assim, a fonte de alimento e propiciando melhores condições para sobrevivência da fauna invertebrada. A umidade atua como fator essencial nas diferentes dinâmicas que acontecem no solo, regulando a disponibilidade de nutrientes na solução do solo além de atuar no metabolismo desses indivíduos (ROSA et al., 2015).

\section{CONCLUSÕES}

Áreas com maior idade de reflorestamento reduz o efeito de borda propiciando menor variabilidade da densidade da fauna invertebrada.

O grupo Symphyla é indicativo de ambientes com estrato arbóreo bem desenvolvido com baixa intensidade luminosa e elevado grau de preservação. Em contraste, os grupos Hymenoptera e Heteroptera são indicativos de ambientes com elevado grau de luminosidade.

A taxa de cobertura do solo e profundidade da serapilheira propicia melhor desenvolvimento da fauna invertebrada da serapilheira.

\section{AGRADECIMENTO}

O presente trabalho foi realizado com apoio da Coordenação de Aperfeiçoamento de Pessoal de Nível Superior - Brasil (CAPES) - Código de Financiamento 001.

\section{REFERÊNCIAS}

AHUATZIN, D. A,; CORRO, E. J.; JAIMES, A. A.; VALENZUELA GONZÁLEZ, J. E.; FEITOSA, R. M., et al. Forest cover drives leaf litter ant diversity in primary rainforest remnants within human-modified tropical landscapes. Biodiversity and Conservation, v. 28, p. 1091-1107, 2019. Disponível em: $<$ https://doi.org/10.1007/s10531-019-01712-z\%20>. doi: 10.1007/s10531-019-01712z\%20

ALMEIDA, H. C.; ALMEIDA, D.; ALVES, M. V.; SCHNEIDER, J.; MAFRA, A. L., et al. Propriedades químicas e fauna do solo influenciadas pela calagem em sistema semeadura direta. Ciência Rural, v.37, n.5, p.1462-1465, 2007. Disponível em: $<$ https://doi.org/10.1590/S0103- 84782007000500040>. Doi: 10.1590/S010384782007000500040 
ALTIVO, F. S.; RODRIGUES, F. C. M. P. Funcionalidade ecológica da restauração de áreas degradadas no bioma mata atlântica, rio de janeiro. Revista Brasileira de Ciências Ambientais (Online), v. 43, p. 17-31, 2017. Disponível em: <https://doi.org/10.5327/Z2176-947820170175>. Doi: 10.5327/Z2176-947820170175

AMPOORTER, E.; BAETEN, L,; VANHELLEMONT, M.; BRUELHEIDE , H.; SCHERER-LORENZEN M., et al. Disentangling tree species identity and richness effects on the herb layer: first results from a German tree diversity experiment. Journal of Vegetation Science, v.26, p.742-755, 2015. Disponível em: <https://doi.org/10.1111/jvs.12281>. Doi: 10.1111/jvs.12281

AZEVEDO, A. D.; CAMARA, R.; FRANCELINO, M. R.; PEREIRA, M. G.; LELES, P. S. S. Estoque de carbono em áreas de restauração florestal da Mata Atlântica. Floresta, v.48, n.2, p.183-194, 2018. Disponível em: <https://dx.doi.org/10.5380/rf.v48i2.54447>. Doi: 10.5380/rf.v48i2.54447.

BARETTA, D.; BROWN, G. G.; CARDOSO, E. J. B. N. Potencial da macrofauna e variáveis edáficas como indicadores da qualidade do solo em áreas com Araucária. Acta Zoológica Mexicana, n.2, p.35-50, 2010. Disponível em: $<$ https://ainfo.cnptia.embrapa.br/digital/bitstream/item/24041/1/AZM-Esp-10-Barettaet-al.pdf>.

BERUDE, M. C.; GALOTE, J. K. B.; PINTO, P. H.; AMARAL, A. A. A mesofauna do solo e sua importância como bioindicadora. Enciclopédia Biosfera, Goiânia, v. 11, n.22, p.14-28, 2015. Disponível em: <http://www.conhecer.org.br/enciclop/2015E/A\%20MESOFAUNA.pdf>.

BIANCHI, M. O.; SCORIZA, R. N.; NOGUEIRA, R.; RESENDE, A. S.; CAMPELLO, E. F. C., et al. Macrofauna Edáfica como Indicadora em Revegetação com Leguminosas Arbóreas. Floresta e Ambiente, v. 24, p.1-8, 2017. Disponível em: <http://dx.doi.org/10.1590/2179-8087.085714>. Doi: 10.1590/2179-8087.085714.

HAMMER; HARPER, D. A. T.; RYAN, P. D. PAST: Paleontological statistics software package for education and data analyses. Paleontological eletronica, 2001. Disponível em: < https://palaeo-electronica.org/2001_1/past/past.pdf>.

INMET - Instituto Nacional de Meteorologia. Disponível em: <http://www.inmet.gov.br/portal/index.php?r=bdmep/bdmep>. Acesso em: 15 ago 2019

KALU, S.; KOIRALA, M.; KHADAKA, U. R. Earthworm population in relation to different land use and soil characteristics. Journal of Ecology and the Natural Environment, v. 7, n.5, p.124-131, 2015. Disponível em: <https://doi.org/10.5897/JENE2015.0511 >. Doi: 10.5897/JENE2015.0511.

KITZ, F.; STEINWANDTER, M.; TRAUGOTT, M.; SEEBER, J. Increased decomposer diversity accelerates and potentially stabilises litter decomposition. Soil Biology and Biochemistry, v.83, p.138-141, 2015. Disponível em: <https://doi.org/10.1016/j.soilbio.2015.01.026>. Doi: 10.1016/j.soilbio.2015.01.026. 
MAAB, S.; CARUSO, T.; RILLIG, M. C. Functional role of microarthropods in soil aggregation. Pedobiologia, v.58, p.59-63, 2015. Disponível em: <http://dx.doi.org/10.1016/ j.pedobi.2015.03.001>. Doi: 10.1016/ j.pedobi.2015.03.001.

MORGADO, R.; FERREIRA, N. G. C.; CARDOSO, D. N.; SOARES, A. M. V. M.; LOUREIRO, S. Abiotic factors affect the performance of the terrestrial isopod Porcellionides pruinosus. Applied Soil Ecology, v.95, p.161-170, 2015. Disponível em: doi:10.1016/j.apsoil.2015.06.012. http://dx.doi.org/10.1016/j.apsoil.2015.06.012. Doi:

MURCIA C. Edge effects in fragmented forests: implications for conservation. Trends in Ecology \& Evolution. v.10, n.2, p.58-62, 1995. Disponível em: <http://dx.doi.org/10.1016/S0169-5347(00)88977-6>. $\quad$ DOI: $10.1016 / S 0169-$ 5347(00)88977-6.

OLIVEIRA, I. R. P.; FERREIRA, A. N.; JÚNIOR, A. B. V.; DANTAS, J. O.; SANTOS, M. J. C., et al. Diversidade de formigas (Hymenoptera; Formicidae) edáficas em três estágios sucessionais de mata atlântica em São Cristóvão, Sergipe. Agroforestalis News, n.1, v.1, p.28-58, 2016. Disponível em: <https://seer.ufs.br/index.php/AGRO/article/view/5387/4635>.

OLIVEIRA FILHO, L. C. I.; BARETTA, D. Por que devemos nos importar com os colêmbolos edáficos? Scientia agraria, v.17, p.21-40, 2016. Disponível em: <http://dx.doi.org/10.5380/rsa.v17i2>. Doi: 10.5380/rsa.v17i2.

OSSOLA, A.; NYMAN, P. Aridity indices predict organic matter decomposition and comminution processes at landscape scale. Ecological Indicators, v.78, p.531-540, 2017. Disponível em: <https://doi.org/10.1016/j.ecolind.2017.03.049>. Doi: 10.1016/j.ecolind.2017.03.049.

PATRIGNANI, A.; OCHSNER, T. E. Canopeo: A powerful new tool for measuring fractional green canopy cover. Agronomy Journal. v. 107, n.6, p.2312-2320, 2015. Disponível em:

https://www.researchgate.net/publication/282353180_Canopeo_A_Powerful_New_T ool_for_Measuring_Fractional_Green_Canopy_Cover>. Doi: 10.2134/agronj $\overline{1} 5.01 \overline{1} 0$.

PEARSON, K. On lines and planes of closest fit to systems of points in space. Philosophical Magazine, v.2, p.559-572, 1901. Disponível em: < https://doi.org/10.1080/14786440109462720>. Doi: 10.1080/14786440109462720.

PETERS, V. E.; CAMPBELL, K. U.; DIENNO, G.; GARCÍA, M.. LEAK, E., et al. Ants and plants as indicators of biodiversity, ecosystem services, and conservation value in constructed grasslands. Biodiversity and Conservation, v.25, p.1481-1501, 2016. Disponível em: <https://doi.org/10.1007/s10531-016-1120-z>. Doi: 10.1007/s10531-016-1120-z.

ROSA, M. G.; FILHO, O. K.; BARTZ, M. L. C.; MAFRA, A. L.; SOUSA, J. P. F., et al. Soil macrofauna and physical and chemical properties under soil management 
systems in the Santa Catarina Highlands, Brazil. Revista Brasileira de Ciência do Solo, v.39, p.1544-1553, 2015. Disponível em: <http://dx.doi.org/10.1590/01000683rbcs20150033>. Doi: $10.1590 / 01000683$ rbcs20150033

SCORIZA, R. N.; CORREIA, M. E. F.; SILVA, E. M. R. Colêmbolos e fungos micorrízicos arbusculares como indicadores de degradação em fragmentos florestais de encosta. Revista de Ciências Agrárias, v.59, n.4, p.386-392, 2016. Disponível em: <http://dx.doi.org/10.4322/rca.2172>. Doi: 10.4322/rca.2172.

SCORIZA, R. N.; CORREIA, M. E. F. Soil Fauna as Indicator in Slope Forest Fragments. Floresta e Ambiente, v.23, n.4, 2016. Disponível em: <http://dx.doi.org/10.1590/2179-8087.135715>. Doi: 10.1590/2179-8087.135715.

SILVA, C. F.; PEREIRA, G. H. A.; PEREIRA, M. G.; SILVA, A. N. Fauna edáfica em área periodicamente inundável na restinga da Marambaia, RJ. Revista Brasileira de Ciência do Solo, v.37, n.3, p.587-595, 2013. Disponível em: <http://dx.doi.org/10.1590/S0100-06832013000300004>. Doi: 10.1590/S010006832013000300004.

SILVA, W. T. M.; LEONARDO, F. A. P.; SOUTO, S. J.; SOUTO, LUCENA, J. D. S.; MEDEIROS NETO, P. H. Deposição de serapilheira em áreas de Caatinga no Núcleo de Desertificação do Seridó. Agropecuária Científica do Semiárido, v.12, n.4, p. 383-390, 2017. Disponível em: <http://dx.doi.org/10.30969/acsa.v12i4.861>. Doi: 10.30969/acsa.v12i4.861.

SOUZA, R. C.; CORREIA, M. E. F.; PEREIRA, M. G.; SILVA, E. M. R.; PAULA, R. $R$., et al. Estrutura da comunidade da fauna edáfica em fragmentos florestais na Restinga da Marambaia, RJ. Revista Brasileira de Ciências Agrárias, v.3, p.49-57, 2008. Disponível em: < http:/www.agraria.pro.br/ojs2.4.6/index.php?journal=agraria\&page $=$ article $\& o p=v i e w \&$ path $\% 5 \mathrm{~B} \% 5 \mathrm{D}=$ agraria_v3i1 a262>. Doi: 10.5039/agraria.v3i1a262

STORK, N. E.; EGGLETON, P. Invertebrates as determinants and indicators of soil quality. American Journal of Alternative Agriculture, Cambridge: Cambridge Journals, v.7, p.38-47, $1992 . \quad$ Disponível em: <https://doi.org/10.1017/S0889189300004446>. Doi: 10.1017/S0889189300004446.

SYLVAIN, Z. A.; WALL, D. H.; CHERWIN, K. L.; PETERS, D. P.; REICHMANN, L. G., et al. Soil animal responses to moisture availability are largely scale, not ecosystem dependent: insight from a cross-site study. Global Change Biology, Nova Jersey, v.20, n.2, p.2631-2643, 2014. Disponível em: < https://www.ncbi.nlm.nih.gov/pubmed/24399762>. Doi: 10.1111/gcb.12522.

TANT, C. J.; ROSEMOND, A. D.; HELTON, A. M.; FIRST, M. R. Nutrient enrichment alters the magnitude and timing of fungal, bacterial, and detritivore contributions to litter breakdown. Freshwater Science, v.34, p.1259-1271, 2015. Disponível em: <https://doi.org/10.1086/683255>. Doi: 10.1086/683255. 
UNDERWOOD, E. C.; FISHER, B. L. The role of ants in conservation monitoring: If, when, and how. Biological Conservation, v.132, p.166-182, 2006. Disponível em: <https://doi.org/10.1016/j.biocon.2006.03.022>. Doi: 10.1016/j.biocon.2006.03.022.

ZARDO, D. C.; CARNEIRO, A. P.; LIMA, L. G.; SANTOS-FILHO, M. Comunidade de artrópodes associada à serrapilheira de cerrado e de mata de galeria, na estação ecológica serra das araras - Mato Grosso do Sul, Brasil. Revista Uniara, v.13, n.2, 2010. Disponível em: <https://doi.org/10.25061/2527-2675/ReBraM/2010.v13i2.143>. Doi: 10.25061/2527-2675/ReBraM/2010.v13i2.143. 